\title{
Afinidades e intercambios: el encuentro entre la Escuela de Bengala y la Bauhaus a través de dos exposiciones (Calcuta 1922-Berlín 1923)
}

\author{
Sol Izquierdo de la Viña \\ Universidad Complutense de Madrid \\ solizquierdo@ucm.es
}

RESUMEN: Dos exposiciones, que tuvieron lugar en un corto intervalo de tiempo, sirven como punto de partida para examinar la red de intercambios artístico-culturales entre la modernidad india y la vanguardia alemana a comienzos de los años veinte. En 1922 una muestra con obras de la escuela de arte Bauhaus fue inaugurada en Calcuta. Al año siguiente, 1923, acuarelas modernas indias se exhibieron en Berlín. En torno a estas dos exposiciones se generó un debate teórico, tanto en la prensa india como alemana, que formuló la modernidad india y proclamó las afinidades entre la vanguardia alemana e india ya en los años veinte, planteando así la existencia de una modernidad mundial.

PALABRAS CLAVE: Bauhaus; Escuela de Bengala; Stella Kramrisch; Modernidad; Teoría Postcolonial; Gaganendranath Tagore; Arte Global.

\section{Affinities and Exchanges: The Encounter between the Bengal School and the Bauhaus through two Exhibitions (Calcutta 1922 - Berlin 1923)}

ABSTRACT: Two exhibitions, which took place within a short space of time, serve as a starting point to study the network of artistic and cultural exchanges between Indian modernism and German avant-garde in the early twenties. In 1922, a major Bauhaus art exhibition was held in Calcutta. In 1923, the following year, modern Indian watercolours were shown in Berlin. These two exhibitions generated a theoretical debate in both the Indian and the German press, in which Indian modernism was formulated and the affinities between Indian and German avant-garde were acclaimed as early as the 1920s, thus establishing the existence of a worldwide modernism.

KEYWORDS: Bauhaus; Bengal School; Stella Kramrisch; Modernism; Postcolonial Theory; Gaganendranath Tagore; Global Art.

Recibido: 28 de febrero de 2018 / Aceptado: 14 de mayo de 2018

\section{Introducción}

Formas geométricas fragmentadas, manchas monocromas, a veces con gradaciones de la misma tonalidad, otras yuxtapuestas a sus complementarios, crean una superficie lumínica y tornasolada que evoca los volúmenes de un templo. Estas líneas concuerdan con el óleo que Lyonel Feininger pintó en 1926 plasmando la vista de la Iglesia de Gelmeroda en Weimar [1], edificio medieval que inspiró innumerables obras, dibujos, grabados y óleos, de este profesor de la Bauhaus. Aunque la descripción se ajusta asimismo a una acuarela del pintor bengalí Gaganendranath Tagore [2] que rememora la voluptuosa forma de montaña del śikhara, el templo hindú típico del norte de India.

Tanto una xilografía de Feininger que representaba la Iglesia de Gelmeroda ${ }^{1}$ como una acuarela de Gaganendranath que plasmaba las formas montañosas de un templo hindú fueron presentadas en las dos exposiciones que tuvieron lugar en India y Alemania. Concretamente, la xilografía del alemán estuvo colgada en una muestra celebrada en Calcuta en 1922,

Cómo citar este artículo: IZQUIERDO DE LA VIÑA, Sol, «Afinidades e intercambios: el encuentro entre la Escuela de Bengala y la Bauhaus a través de dos exposiciones (Calcuta 1922-Berlín 1923)", Boletín de Arte-UMA, n. ${ }^{\circ}$ 39, Departamento de Historia del Arte, Universidad de Málaga, 2018, pp. 125-138, ISSN: 0211-8483, DOI: http:// dx.doi.org/10.24310/BoLArte.2018.v0i39.4234 


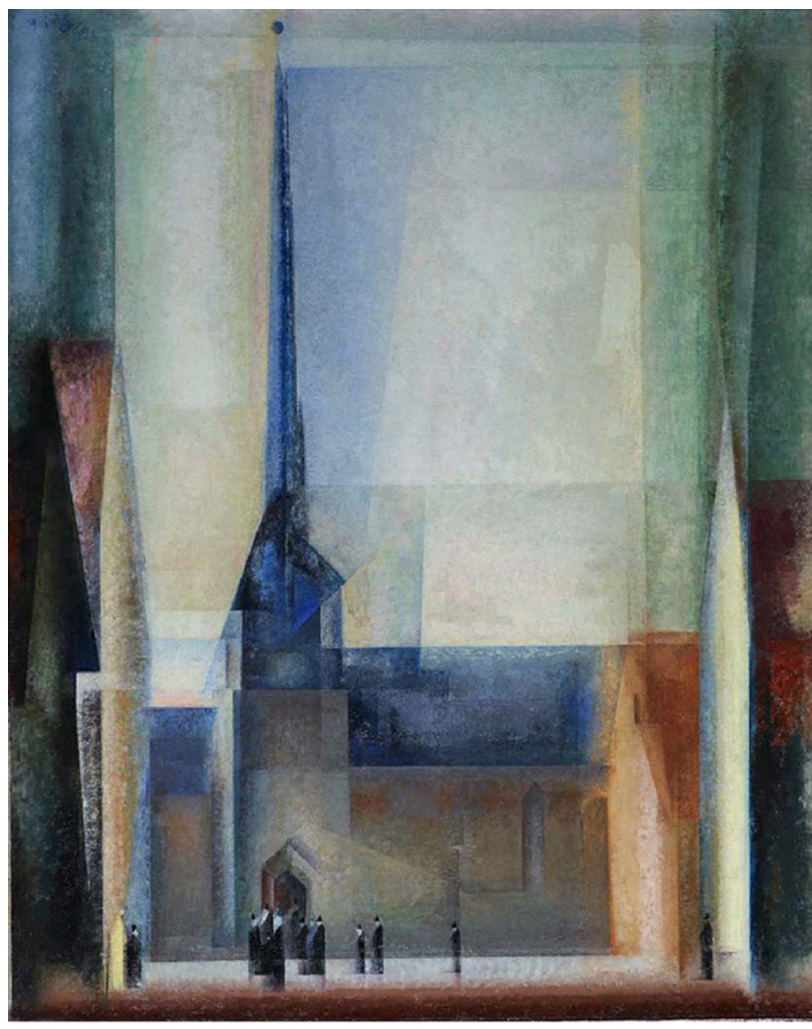

1. Lyonel Feininger, Gelmeroda IX, 1926. Óleo sobre lienzo, 100 x $80 \mathrm{~cm}$, Folkwang Museum, Essen, Alemania. @ VG Bild-Kunst, Bonn 2014

en la que se expusieron obras en papel de los profesores y alumnos de la escuela de arte Bauhaus, constituyendo la primera exhibición de la vanguardia europea en India. Mientras que la acuarela del indio pudo verse en una exposición inaugurada en Berlín en 1923, en la que se presentó una selección de acuarelas modernas bengalíes por primera vez en Alemania. Se trataba de obras realizadas principalmente por los pintores asentados en Calcuta, que formaban la denominada Bengal School, y algunas de los maestros y discípulos de la escuela de arte Kala-Bhavana, que había sido recientemente fundada en Śāntiniketan, en el interior rural de Bengala Occidental. Estos pequeños folios constituyen el testimonio de la experimentación estética que devino en un fructífero intercambio transcultural entre artistas e intelectuales quienes, a pesar de la enorme distancia que les separaba, compartían intereses afines.

La red de intercambios que culminó en estas dos exposiciones y el debate teórico surgido en torno a ellas, son investigados con el fin de tratar diversas cuestiones respecto a la configuración de la modernidad india y su formulación teórica, así como su vinculación con la vanguardia alemana y su recepción en este país. Se abordan, por lo tanto, problemáticas propias de una aproximación postcolonial, fundamentalmente la existencia de múltiples núcleos de vanguardia que supera la noción centro/periferia heredada de las relaciones de poder de la época colonial. La hipótesis de una relación con intereses recíprocos entre diversos centros de una supuesta modernidad global, trata de demostrarse no sólo tomando la crítica postcolonial como referencia, sino arguyendo que ya en las teorías expuestas a principios de los años veinte es patente un interés consciente por borrar las fronteras y encontrar los puntos comunes entre el arte indio y el europeo ${ }^{3}$.

Esencial para este estudio es la revisión de la modernidad y pre-modernidad artística en India realizada por Partha Mitter, quien trata de eliminar de su narración las relaciones jerárquicas que han envuelto los discursos sobre el arte no occidental (Mitter, 1994; 2007; 2008; 2013). Se evitan asimismo conceptos como el de «influencia estilística» para dar paso a términos propios de la teoría postcolonial como hybridity (hibridación), acuñado por Homi K. Bhabha (Bhabha, 1994; Struve, 2013: 97-117), que permiten una valoración estética de las obras nacidas en un contexto transcultural. Entretanto, se tienen en cuenta como pilares para la investigación los diversos actores, instituciones y medios (principalmente revistas y catálogos) que conformaron el entramado que promovió la realización de las dos exposiciones. El objetivo es iluminar el proceso de la modernidad mundial a través de ejemplos concretos y locales, insertos en dinámicas transregionales, en lugar de aportar una visión global homogeneizadora.

Exclusivamente dos publicaciones recientes se ocupan del tema investigado. En primer lugar, el catálogo de una exposición que documentaba el intercambio que supuso la exposición de la Bauhaus en Calcuta y su repercusión en la escuela, publicado por Regina Bittner y Katherin Rhomberg en 2013 con el título Das Bauhaus in Kalkutta. Eine Begegnung kosmopolitischer Avantgarden. En segundo lugar, el estudio sobre el intercambio intelectual que se produjo entre Alemania e India desde el siglo XIX hasta la Segunda Guerra Mundial llevado a cabo por Kris K. Manjapra en Age of Entanglement. German and Indian Intellectuals Across Empire 
(2014), único autor que se ha ocupado de la exposición de Berlín hasta el momento. No obstante, la base fundamental de la investigación está constituida por fuentes primarias, los catálogos de ambas exposiciones (Kramrisch, 1922d; Kumar Sarkar, 1923) así como artículos publicados en revistas y periódicos, principalmente en el journal bengalí de arte oriental Rupam ${ }^{4}$.

\section{Dos exposiciones como encrucijada}

\section{La Bauhaus en Calcuta (1922)}

El 23 de diciembre de 1922 se inauguró la Decimocuarta Exposición Anual de la Indian Society of Oriental Art en Calcuta. Junto con las obras de los artistas locales se expuso una selección de trabajos de la escuela de arte Bauhaus que constituyó la primera exhibición de la vanguardia europea en India ${ }^{5}$.

La exposición de la Bauhaus fue concebida y organizada por la historiadora del arte austriaca Stella Kramrisch (1896-1993)6 , que había llegado a India en 1921 gracias a la invitación de Rabindranath Tagore (1861-1941). El poeta y filósofo indio, que había conocido a Kramrisch durante una conferencia en Oxford en 1920, le propuso formar parte de su proyecto pedagógico, emprendido en la Universidad Visva-Bharati situada en Śāntiniketan, en el interior rural de Bengala (Stoler Miller, 1983: 8-9)7. Asimismo, Tagore visitó Weimar en 1921, cuya presencia fue transcendental para algunos miembros de la recién fundada Bauhaus (Friedewald, 2013: 122; Mitter, 2007: 17) ${ }^{8}$. La internacionalidad de Tagore fue clave para el desarrollo de la exposición, pero fue Kramrisch quien escribió una carta ${ }^{9}$ a Johannes Itten (18881967) proponiendo la realización de la muestra en Calcuta y quien redactó el texto para el catálogo (Manjapra, 2011: 34-40) ${ }^{10}$. Una fotografía de la inauguración de la exposición en Calcuta testimonia la presencia de la historiadora vienesa entre los miembros de la Indian Society of Oriental Art de Calcuta [3] (Bittner/Rhomberg, 2013: 76-80).

En Calcuta se expusieron ciento setenta y cinco obras en papel de alumnos y profesores de la Bauhaus, entre las que sobresalen obras de Johannes Itten, Lyonel Feininger (1871-1956), Wassily Kandinsky (1866-1944) y Paul Klee (1879-1940) (Manjapra, 2014: 247). Desde Weimar se envió

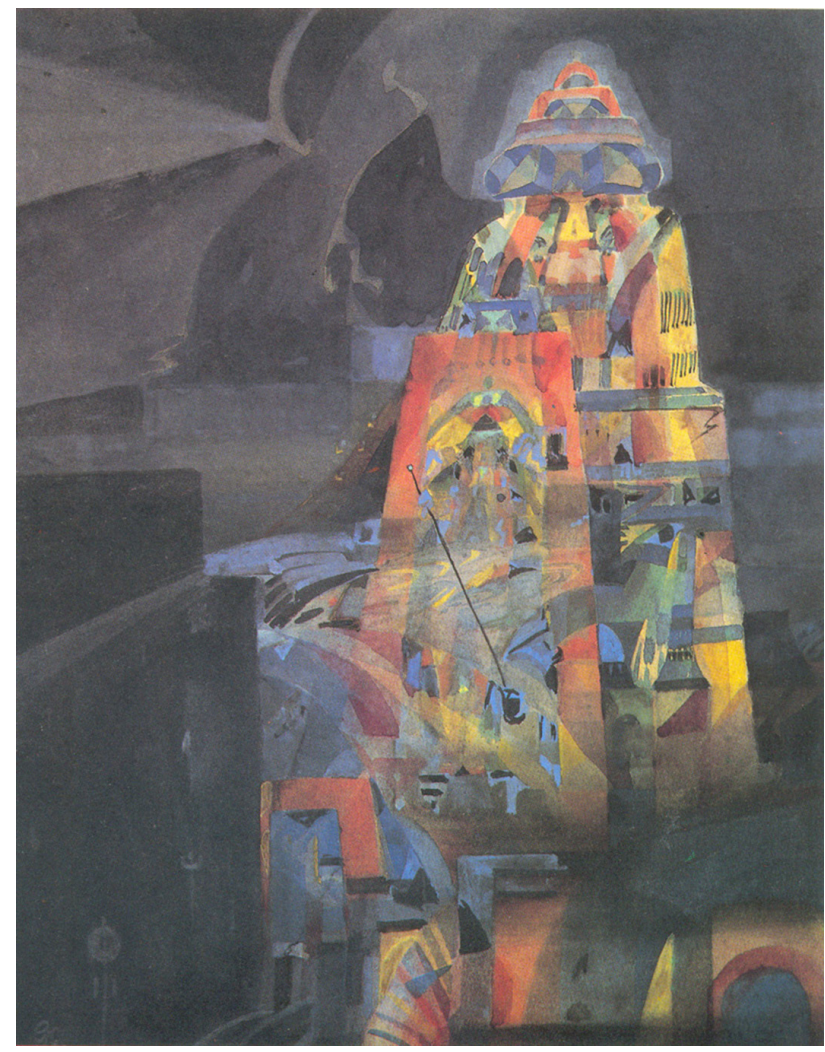

2. Gaganendranath Tagore, Tempel, ca. 1920-1925. Acuarela sobre papel, National Gallery of Modern Art, New Delhi, India

también el libro Utopia. Dokumente der Wirklichkeit, publicado en 1921, que había sido confeccionado por Bruno Adler e Itten, y reunía antiguos textos filosóficos y religiosos, entre los que destaca un fragmento del Rigveda sobre la creación (Friedewald, 2013: 130)11. La muestra de obras europeas e indias al alimón, fue un evento sin precedentes en la cosmopolita ciudad de Calcuta, mas su transcendencia reside además en su carácter pionero, pues suponía simultáneamente un ensayo internacional de las ulteriores exhibiciones de la Bauhaus, ya que la primera gran exposición pública de la escuela fue realizada en el verano de 1923 en Weimar (Foster, 2004: 185). Desde 1919 hasta 1925 la escuela de arte Bauhaus estuvo asentada en la ciudad de Weimar, aunque dirigida por Walter Gropius, el establecimiento del curso preliminar Vorkurs impartido por Johannes Itten llevó a que su espíritu místico y su ideario utópico impregnaran durante estos primeros años el ambiente de la escuela. Itten, profun- 


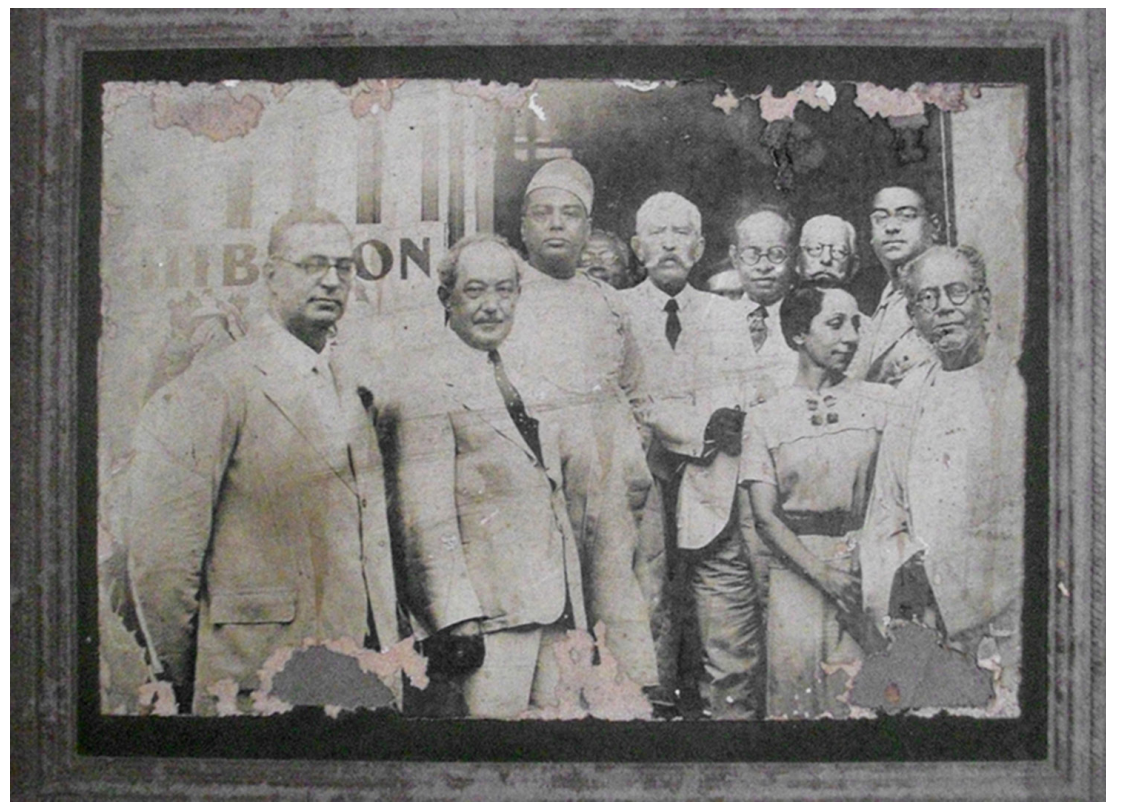

3. Stella Kramrisch con los miembros de la Indian Society of Oriental Art, Calcuta, 1922. Autor desconocido, Journal of the Indian Society of Oriental Art, 1981, Fotografía (C) Fundación Bauhaus Dessau, Alemania

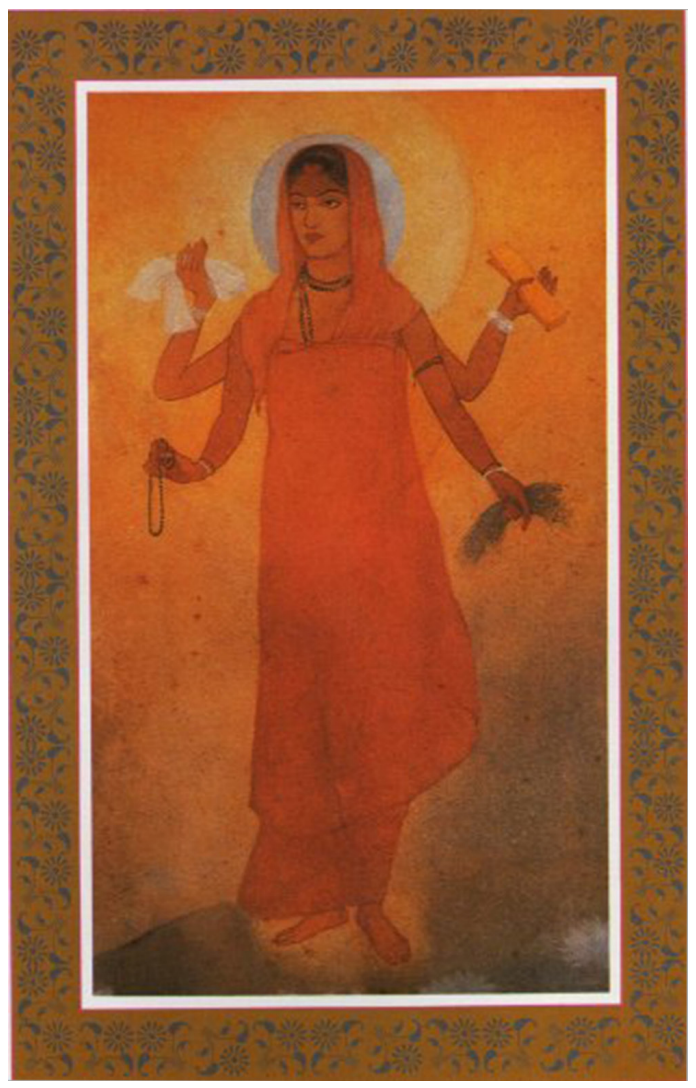

4. Abanindranath Tagore, Bharat Mata (Mother India), 1905. Acuarela sobre papel, $10,5 \times 6 \mathrm{~cm}$, Victoria Memorial Hall, Kolkatta, India damente interesado por diversas corrientes esotéricas como la Teosofía y el Mazdeismo, dejó la escuela en 1923 para vivir en una colonia mazdeista en Suiza. A partir de entonces, a la actitud utópica de Itten y Adler sucedió una visión más pragmática y funcional que abogaba por la unidad entre arte y tecnología; transformación inexorable tras el traslado de la Bauhaus a Dessau en 1925 (Wagner, 2005: 65-74).

Las obras indias contemporáneas exhibidas junto a las alemanas, procedían principalmente de la Escuela de Bengala (o Bengal School), corriente pictórica originada en Calcuta en torno a la Indian Society of Oriental Art, que había sido fundada en 1907 por los hermanos Abanindranath Tagore (1871-1951) y Gaganendranath Tagore (1867-1938) ${ }^{12}$. Estos artistas promulgaban la eliminación de los principios formales naturalistas impuestos por la academia occidental, mientras abogaban por la renovación del arte indio a través de la recuperación de las antiguas tradiciones, principalmente la miniatura mogola, con el fin de configurar un estilo nacional, que ansiaba incluso superar las fronteras indias en pos del Panasianismo. La obra más distintiva del movimiento es Bharat Mata (1905) de Abanindranath Tagore [4] que representa a la "Madre India». La Escuela de Bengala produjo, en algunos casos, un estilo ecléctico y en otros, experimentaciones formales afines a la vanguardia occidental (Guha-Thakurta, 1992; Mitter, 1994). 
5. Johannes Itten, (Komposition) Komposition III, página 9 de la carpeta 10 Originallithographien, 1919.

Litografía sobre papel, $60 \times 45 \mathrm{~cm}$, Bauhaus-Archiv Berlin, Alemania (Bittner/Rhomberg, 2013: 35)

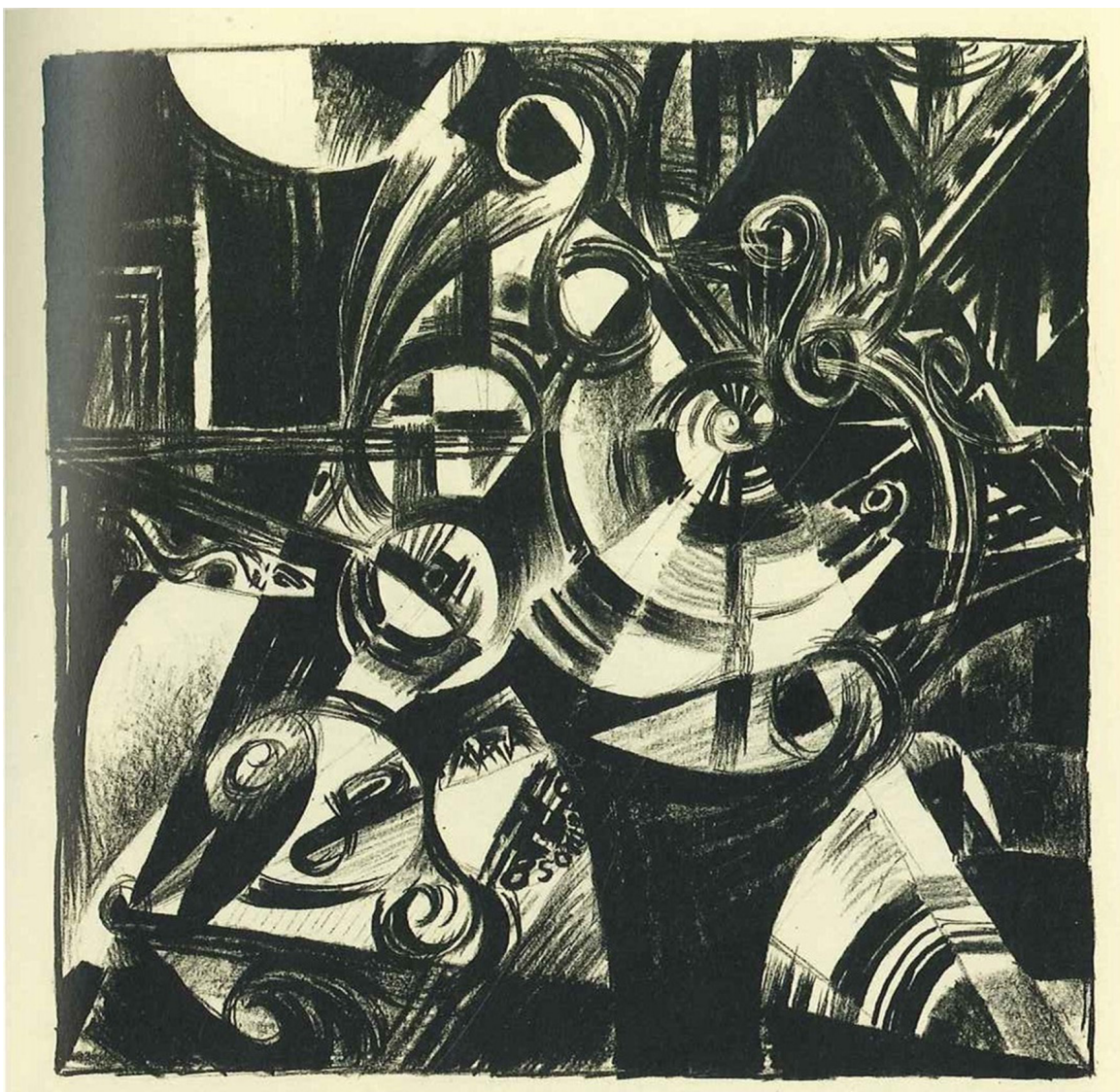

El público bengalí pudo contemplar obras como Komposition III (1919) de Johannes Itten [5], profesor de la Bauhaus fascinado por la filosofía y las tradiciones orientales, junto con los denominados primeros ensayos «cubistas» de Gaganendranath Tagore que vieron la luz aquel mismo año, como Artist at Jorasanko House [6]. De este modo, se configuró por primera vez un espacio para la comparación entre la vanguardia europea e india ${ }^{13}$. La comisaria de la exposición, Stella Kramrisch, apela en su texto para el catálogo al parangón entre ambas vanguardias. En dicho texto Kramrisch hizo un Ilamamiento al público indio, para que discerniese que el arte europeo no era únicamente el impuesto por las academias del régimen colonial, sino que también existían en Europa tendencias que rechazaban el naturalismo académico en busca de la «expresión». Así, la historiadora vienesa concluye su redacción del catálogo con la siguiente aserción:

\begin{abstract}
El público indio debería estudiar esta exposición, así aprendería que el Arte Europeo no significa «naturalismo» y que la transformación de las formas de la naturaleza en la obra de un artista es común a la India moderna y antigua y a Europa, como una expresión inconsciente y, por lo tanto, inevitable, de la vida del alma y del genio artístico (Kramrisch, 1922d: 11-14) $)^{14}$.

El año 1922 simboliza, en efecto, el punto de irrupción de la modernidad en India, tal y como ha señalado por Partha Mitter ${ }^{15}$ pues, aunque ésta se venía fraguando desde hacía tiempo, la exposición de la Bauhaus implicó cambios decisivos para su configuración. Por un lado, se rechazó definitivamente el gusto por el naturalismo de herencia colonial (Mitter, 2007: 15) y, por otro, supuso la internacionalización del arte moderno indio (Manjapra, 2011: 37), que había sido expuesto al público con el mismo estatus que el arte euro-
\end{abstract} peo de vanguardia. 


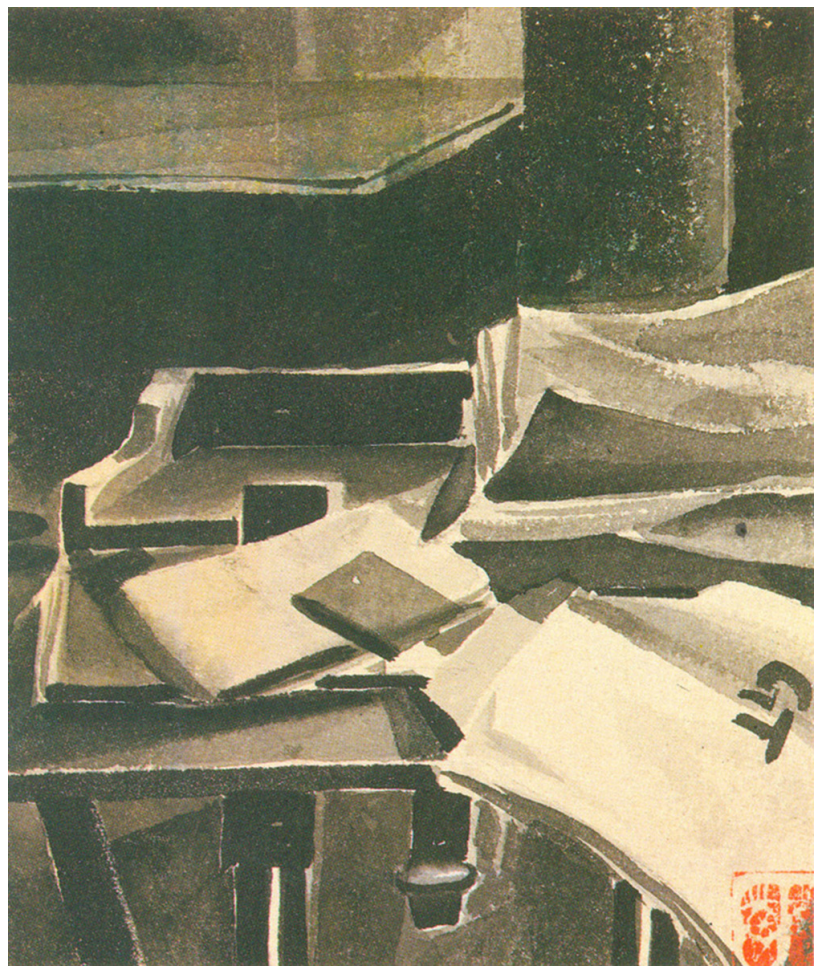

6. Gaganendranath Tagore, Artist at Jorasanko House, sin fecha. Tinta y lavada sobre papel, $11,2 \times 14 \mathrm{~cm}$, National Gallery of Modern Art, New Delhi, India. (Bittner/Rhomberg, 2013: 45)

Acuarelas modernas indias en Berlín (1923)

Pocos meses después, el 2 de febrero de 1923, se inauguró la exposición de acuarela moderna india en el Kronprinzenpalais, edificio de la Nationalgalerie de Berlín. El sociólogo y economista indio Benoy Kumar Sarkar (1887-1949) ${ }^{16}$ propuso la realización de la muestra titulada Ausstellung moderner indischer Aquarelle, que fue organizada con ayuda del director de la galería, el célebre historiador del arte Ludwig Justi (1876-1957) (Kumar Sarkar, 1923).

El Kronprinzenpalais se instituyó en 1919 como sección de la Galería Nacional berlinesa dedicada al arte moderno bajo la rúbrica Galerie der Lebenden (Galería de los vivos). Como primer museo estatal dedicado al arte contemporáneo en Alemania, fue una pieza clave en la institucionalización del arte de vanguardia durante la República de Weimar, principalmente del denominado Expresionismo (Winkler, 2002: 83-86). En este contexto fue organizada la exposición de acuarelas indias, inaugurada tras otra de Paul Klee, constituyendo la primera muestra de arte no europeo realizada en la galería (Janda/Grabowski, 1992: 70-71); si bien las obras de los pintores de Bengala habían sido expuestas en 1914 en Europa, tanto en el Grand Palais de París como en el Victoria and Albert Museum de Londres (Kumar Sarkar, 1923: s.n.).

Ciento trece obras de artistas bengalíes fueron expuestas en Berlín. Entre los cuarenta y seis artistas presentes cabe destacar a Gaganendranath Tagore, Abanindranath Tagore y Rabindranath Tagore, Nandalal Bose (1882-1966) y Sunayani Devi (1875-1962). En el catálogo de la muestra, Benoy Kumar Sarkar trata de explicar el origen y las características de las obras indias al público alemán, subrayando su labor de recuperación de las tradiciones originarias indias, impulsada por una «conciencia nacional» (Kumar Sarkar, 1923: s.n. $)^{17}$. No obstante, concluye defendiendo la certidumbre de "cierta unidad espiritual que conecta de manera orgánica todo el mundo del arte moderno» (Kumar Sarkar, 1923: s.n. $)^{18}$, considerando así la existencia de una modernidad global.

Pocos testimonios han quedado de los motivos que condujeron a la organización de esta muestra. En la portada del catálogo se indica la colaboración tanto de la Indo-europäische Handelsgesellschaft (Indio-Europa Trading Company) como de la Deutschen morgenländischen Gesellschaft, dos compañías dedicadas al intercambio comercial internacional, la primera impulsada por indios y la segunda por alemanes (Kumar Sarkar, 1923). Esto lleva a sospechar acerca de una intención económica subyacente en la realización de este evento, algo que concuerda tanto con la Kulturpolitik de la República de Weimar como con los intereses del sociólogo y economista indio que redactó el texto para el catálogo. Benoy Kumar Sarkar, que compartía con ciertos economistas alemanes la creencia de que la hegemonía del Imperio Británico estaba llegando a su fin y el interés por constituir un nuevo orden de intercambios comerciales internacionales ajenos a éste, se dedicó desde la Indo-europäische Handelsgesellschaft a mejorar el diálogo transnacional y las relaciones anticoloniales de India, como indica Manjapra (2014: 149-155)19. Así, probablemente, la realización de la exposición formó parte de este proyecto.

Incluso en la prensa se destacó que «los hilos» que conectaban Alemania e India eran cada vez más fuertes y 
se mencionaron las relaciones impulsadas por la Indo-europäische Handelsgesellschaft, que desde 1921 se había instaurado en Berlín, argumentando que «primero van los comerciantes, los artistas les siguen; esto es costumbre antigua desde hace milenios» (Osborn, 1923a). No obstante, no hay que poner en duda el interés de los intelectuales y artistas alemanes en el arte indio contemporáneo, aunque no se conozcan con exactitud los pormenores sobre la puesta en marcha de la exposición de Berlín. Pues la realización de la muestra en el Kronprinzenpalais supuso la institucionalización del arte indio moderno en el mismo plano que la vanguardia europea, también en Occidente.

\section{Diálogo teórico en torno a las exposiciones}

En paralelo a las dos exposiciones se desencadenó un acalorado debate teórico, por un lado en Rupam, la revista de la Indian Society of Oriental Art y, por otro, en la prensa alemana, donde se publicaron testimonios de la muestra en Berlín y de los contactos con la cultura india. Estos textos dan cuenta de la relación entre la modernidad india y la vanguardia europea a principios de los años veinte.

En Rupam se produjo un diálogo intelectual en torno a la cuestión de la modernidad india que involucró principalmente a los dos comisarios, Benoy Kumar Sarkar y Stella Kramrisch. Sarkar inició el debate con el artículo «The Aesthethics of a Young India», escrito desde París en 1922 (Kumar Sarkar, 1922: 8-24). El sociólogo criticaba la vuelta a la tradición india, que se había producido como rechazo al naturalismo impuesto por las academias coloniales, mientras que abogaba por un acercamiento a la modernidad occidental. Cuestiones estéticas y políticas se mezclan en su discurso que apela a la noción moderna de «autonomía estética» aunque asociada a la demanda independentista de autogobierno en India bajo la premisa «shilpa as a svaraja in itself» (arte como autogobierno) (Kumar Sarkar, 1922: 16)²; siguiendo la terminología acuñada por el Movimiento de Independencia indio. Pretendía, por lo tanto, a través de la autonomía formal, la liberación del arte indio tanto del contenido literario como histórico-nacionalista, para alcanzar así la emancipación político-cultural y estética, y por consiguiente artística. Cabe considerar en este punto el pensamiento defendido por el ilustre historiador del arte indio Ananda K.
Coomaraswamy quien en su artículo Art and Swadeshi (ca. 1910) se expresaba en un sentido análogo, defendiendo el poder del arte para la emancipación india con las siguientes palabras: «Tratemos de creer en la regeneración de India a través del arte, y no únicamente por medio de la política y la economía» (Coomaraswamy, 1994: 3)21.

Por su parte Kramrisch, quien admiraba la vitalidad del arte indio tradicional, contestó al artículo de Sarkar desde India a través de otro en la misma revista. En él, reprobaba la concepción europeizadora del sociólogo indio. Pues para ella, la expresión artística procedía de la unión entre la intuición personal y el contexto histórico del artista, por lo que la vuelta a la tradición debía desempeñar un papel transcendental en la configuración del arte moderno indio ${ }^{22}$.

En estos textos, cimiento ideológico de las dos exposiciones, queda patente el interés por la formulación teórica de la modernidad india, en la que Sarkar y Kramrisch representaron un rol primordial. La vinculación con la tradición india, por un lado, y con las corrientes europeas contemporáneas por otro, se erigen como controversias cardinales para la configuración de la modernidad india. Tanto las dos exposiciones como la publicación de estos artículos, coinciden con una coyuntura gozne para el arte moderno indio, en la que se puso en tela de juicio el aislamiento y eclecticismo del arte nacional de la Escuela de Bengala ${ }^{23}$, y comenzaron a realizarse experimentos artísticos en la escuela de arte Kala-Bhavana de la Universidad fundada por Rabindranath Tagore en Śāntiniketan²4.

En Alemania se publicaron igualmente numerosos artículos que testimonian la inauguración de la exposición en Berlín. Muchos de ellos fueron recogidos, traducidos y publicados en la revista Rupam, como por ejemplo en «The Indian Art Exhibition: Cuttings from German Press» (1923), lo que evidencia el interés de los intelectuales indios por la recepción del arte nacional en Occidente. La fascinación por la cultura india en Alemania se remonta al «Orientalismo» de los siglos XVIII y XIX, si bien en la República de Weimar se produjo una efervescencia de intercambios directos (Oesterheld, 2014: 101-114). En el caso de la figura de Rabindranath Tagore se advierte un eco mesiánico al rastrear las referencias a India en los periódicos de la época ${ }^{25}$.

El crítico alemán Max Osborn (1870-1946) publicó reseñas de la exposición en el periódico Vossische Zeitung ${ }^{26}$, traducidas posteriormente en Rupam. El crítico se refiere a 
las obras expuestas como «documentos de una grandiosa lucha por la libertad cultural» (Osborn, 1923a) ${ }^{27}$. Elogia la obra de Abanindranath Tagore y Gaganendranath Tagore, a quien compara con los pintores expresionistas con las siguientes palabras: «Gogonendra Nath Tagore [sic.] parece haber incorporado inspiración cubista. El folio en blanco y negro de un Templo armoniza lo asiático con las formas cristalinas, como las que Feininger emplea con gusto. La preciosa fantasía de colores tornasolados En el país de las aves se acerca a un Franz Marc» (Osborn, 1923b: 1)28. En este mismo sentido proclama la afinidad entre las vanguardias internacionales: «El anhelo mundial por una gran expresión se hace evidente aquí - el anhelo que hoy se ha apoderado de todos los artistas de todos los países y razas» (Osborn, 1923c: 77$)^{29}$.

También Kramrisch insistió en los vínculos entre el arte europeo y el indio, y sostuvo la idea de una inspiración mutua de Occidente en Oriente y viceversa. Es su artículo «Indian art and Europe» (1922), describía la relación recíproca entre la cultura occidental y oriental, y sostenía que la correspondencia entre ambos residía en una "afinidad interior» o condiciones culturales similares (Kramrisch, 1922b) ${ }^{30}$. Kramrisch toma como ejemplo la pintura de Gaganendranath Tagore para desentramar los flujos de inspiración. En su artículo «An Indian Cubist» (1922) advierte la experimentación del pintor cercana al «cubismo» y señala:

El Cubismo fue un «descubrimiento» de Occidente. Sus raíces, sin embargo, se encuentran en Oriente. Los fondos de las pinturas en Ajanta, muchos objetos representados en Bharut y Sanchi, la arquitectura en las pinturas Rajput, son visualizadas de una manera cubista. El arte moderno europeo, cansado de la reproducción [mimética], emprende el redescubrimiento de la sugestión expresiva de los elementos primarios del arte, de la línea, el cubo y sus relaciones, de los colores y su vida, y de las luces y sombras (Kramrisch, 1922c: 108$)^{31}$.

Las pequeñas acuarelas y tintas cubistas realizadas por Gaganendranath Tagore desde los primeros años de la década de los veinte [7], constituyen una fracción de su fecunda y polifacética obra, y han sido un caso de estudio paradigmático en el contexto de las problemáticas centro/ periferia. Si bien, como queda patente en la crítica de Os- born, en los años veinte fueron parangonadas con las obras de la vanguardia alemana sin ser por ello menospreciadas, mientras a finales de los cincuenta fueron tildadas despectivamente de «híbrido colonial» por W. G. Archer en su libro India and Modern Art (1959). Pues como ha puesto de manifiesto Partha Mitter bajo el lema de «The Picasso Manqué syndrome", siguiendo la dialéctica del discurso jerarquizado en centro/periferia, los préstamos del arte africano no han comprometido la originalidad de artistas de la cultura dominante como Picasso, mientras que la utilización de formas cercanas al cubismo ha hecho tambalear no sólo la modernidad de la obra de Gaganendranath, sino también su integridad y carácter indio (Mitter, 2008: 534-537).

Considero relevante destacar al respecto que ya en los años veinte en la revista Rupam se puso en tela de juicio el concepto de «influencia estilística». En el artículo «European Influence on Modern Indian Art» (1922) se realiza una vehemente crítica al uso de este término, que en Occidente ha sido cuestionado de nuevo por la teoría postcolonial: «EI quid de la cuestión radica en una síntesis intelectual y espiritual de las culturas oriental y occidental, en la cual la cuestión de la "influencia" es un tema irrelevante y engañoso. La palabra "influencia" inevitablemente sugiere una dominación o la supremacía de uno sobre el otro» (Rupam, 1922: 109)32.

De esta manera, se pone de relieve cómo ya en las teorías formuladas en los años veinte es palmario el afán por revisar los conceptos tradicionales de los discursos hegemónicos, así como el deseo por tender los lazos entre las corrientes orientales y occidentales. Corresponde al convencimiento de lo que Sarkar denominó «cierta unidad espiritual que conecta de manera orgánica todo el mundo del arte moderno" y Kramrisch definió como "afinidad interior»; enunciados que evidencian la concepción de la modernidad como un fenómeno mundial ya en aquel momento.

\section{Conclusiones: confluencias entre las modernidades india y alemana}

En forma de epílogo es posible trazar un mapa de los caminos confluyentes entre las corrientes artísticas alemana e india, que desembocaron en el encuentro que cristalizó en las dos exposiciones. La «búsqueda de lo espiritual» en la que el arte se erige como adalid contra el excesivo de- 
sarrollo material del mundo moderno, se establece como idea inicial que interconectó ambas culturas. En Occidente, la crisis espiritual finisecular se nutrió del pensamiento indio para conformar nuevas teorías como la Teosofía, que a su vez tuvo una importancia decisiva para los artistas de vanguardia que realizaron las primeras pinturas abstractas como Wassily Kandinsky, lo que quedó reflejado en su obra teórica Über das geistige in der Kunst (1911). La relevancia de la religión india para la creación moderna occidental se manifiesta también en el ya citado Utopia. Dokumente der Wirklichkeit (1921). Así, la Bauhaus de Weimar funcionó como crisol de estas ideas místicas que obsesionaron a los artistas europeos.

Simultáneamente, la noción de que India era el limen espiritual mundial, fue también detentada por los intelectuales indios como Ananda K. Coomaraswamy y el círculo constituido alrededor de la familia Tagore a comienzos de siglo en Calcuta. La «espiritualidad» se convirtió por ello en el caballo de batalla de la configuración del arte nacional indio que fue plasmado por los artistas de la Escuela de Bengala, lo cual supuso un primer ensayo moderno, al rechazar el naturalismo impuesto por las academias y el desarrollo material producto de la colonización. Como queda reflejado a lo largo del artículo, el interés fue mutuo, pues Kandinsky e Itten estaban tan fascinados por la filosofía india como Gaganendranath por la experimentación formal de sus coetáneos europeos.

El siguiente hito en el mapa se localiza en 1919, año en el que fueron fundadas la Bauhaus en Weimar y la Kala-Bhavana en Śāntiniketan. Dos utopías pedagógicas que con una visión holística y social promovieron la renovación de la cultura material y del entorno se instauraron al mismo tiempo en Alemania y en India. Como es sabido, la Bauhaus de Weimar se caracterizó por la defensa del diseño moderno y de la artesanía todavía intacta en las culturas no industrializadas. Mientras tanto en India, el deseo por recuperar las técnicas artísticas tradicionales se fundamentaba en la búsqueda de la identidad nacional y en la estrategia Swadeshi del Movimiento de Independencia indio que promulgaba el boicot a los productos ingleses en pos de la autosuficiencia.

Así, el óleo de Feininger Gelmeroda IX [1] y la acuarela de Gaganendranath Templo [2] simbolizan las afinidades entre la vanguardia alemana y la modernidad india. Pues a pesar de las diferencias entre ambas obras, que se advierten

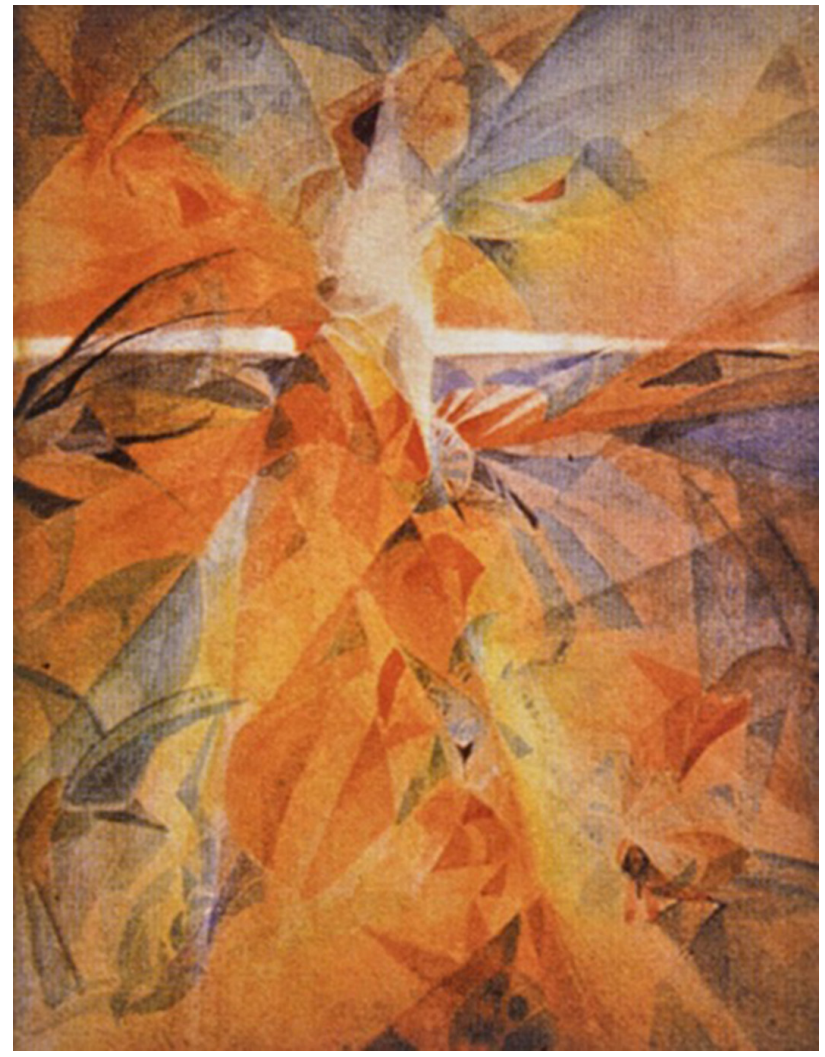

7. Gaganendranath Tagore, Rabindranath Tagore in the Island of Birds, 1921-1925. Tempera sobre papel, $17 \times 21,2 \mathrm{~cm}$, National Gallery of Modern Art, New Delhi, India

en las formas puntiagudas y la tonalidad fría del primero, que contrastan con la voluptuosidad de las formas redondeadas y la predominancia de los colores cálidos del segundo. El templo, ya sea la iglesia medieval cristiana o el śikhara hindú, se erige en ambos como cimiento tradicional y al mismo tiempo como contrafuerte espiritual de estas corrientes vanguardistas que trataron de subvertir las normas impuestas por la academia a través de la experimentación formal, así como luchar contra el excesivo desarrollo material producido por la industrialización y expandido a través del colonialismo.

El intercambio directo entre la modernidad india y la vanguardia alemana se hizo efectivo en torno a estas dos exposiciones celebradas en Calcuta en 1922 y en Berlín en 1923. Ambas fueron fruto del interés consciente y recíproco por impulsar los intercambios culturales entre India y Alemania. Debe interpretarse este encuentro como una correspon- 
dencia o afinidad entre dos centros de la modernidad global, conclusión que deviene de una revisión historiográfica y terminológica, que se sustenta en la crítica postcolonial, pero que tiene como fundamento principal las fuentes de la épo- ca. Las exposiciones de Calcuta y Berlín encarnan no sólo una encrucijada entre los caminos del avance de la vanguardia artística india y alemana, sino también la coyuntura crítica en la que irrumpió la emboscada de la modernidad mundial.

\section{Notas}

1 Referencia de la obra: Lyonel Feininger, Gelmeroda, 1920, xilografía sobre papel japonés, 33,1 x 24,5 cm, Bauhaus-Archiv Berlin (Bittner/Rhomberg, 2013: 31).

2 Referencia de la obra: Gaganendranath Tagore, Der Tempel, № 99 en el catálogo de la exposición de Berlín que se encuentra en el archivo del Ibero-Amerikanisches Institut de Berlín (Kumar Sarkar, 1923: s.n.).

3 Esta investigación tiene su origen en el verano de 2014, iniciada como trabajo para un seminario impartido por el Profesor Jyotindra Jain (Jawaharlal Nehru University, Delhi), invitado en la Humboldt Universität de Berlín, realizado en el marco de los estudios de Master de Historia del Arte en el Contexto Global en la Freie Universität Berlin. Para la redacción de este artículo he contado con el consejo de Sergio Román Aliste, investigador del grupo UCM TRAMA que ha realizado su tesis doctoral sobre la vinculación de la enseñanza artística y el arte moderno en la escuela de arte Kala-Bhavana en Śāntiniketan, Bengala Occidental, India (Román Aliste, 2015). La finalización del artículo ha sido posible gracias a un contrato predoctoral UCM-Santander en el Departamento de Historia del Arte de la Universidad Complutese de Madrid.

4 Las fuentes consultadas se encuentran en los archivos de la Staatsbibliothek de Berlín y del Ibero-Amerikanisches Institut de Berlín.

5 Véase la reseña sobre la exposición: «The Fourteenth Annual Exhibition of the Indian Society of Oriental Art» (1923), Rupam, No. 13-14, January-June, pp. 14-18. Rupam: An illustrated Quarterly Journal of Oriental Art, chiefly Indian, fue la revista de la Indian Society of Oriental Art, editada por Ordhendra C. Gangoly y publicada en Calcuta entre 1920 y 1930, aproximadamente.

6 La figura de Stella Kramrisch ha comenzado a ser valorada en los últimos años a la luz de los estudios transculturales. Historiadora del arte indio, formada en la Universidad de Viena, realizó su tesis doctoral sobre escultura budista con Joseph Strzygowski (quien detentó el primer puesto como profesor de arte oriental en una universidad europea). Kramrisch tuvo, al mismo tiempo, gran interés por las corrientes artísticas contemporáneas y participó en la vida intelectual de Viena. Es relevante destacar que fue la primera europea que escribió sobre arte contemporáneo indio (Stoler Miller, 1983: 3-33).

7 Como profesora invitada por Rabindranath Tagore en la Universidad de Śāntiniketan, Stella Kramrisch impartió lecciones de Historia del Arte entre 1921 y 1923. En 1923 se trasladó a la Universidad de Calcuta, donde fue profesora hasta los años cuarenta (Bittner/Rhomberg, 2013: 78).

8 La visita de Tagore fue relatada por los periódicos alemanes, ya que el poeta realizó un recital en el Deutsches Nationaltheater de Weimar; tuvo especial importancia para Johannes Itten.

9 Véase las reproducciones de las cartas de Kramrisch a Itten de 1922 (Bittner/Rhomber, 2013: 69).

10 K. Manjapra fue el primero en afirmar el rol fundamental que Stella Kramrisch tuvo en la concepción y organización de la exposición de obras de la Bauhaus en Calcuta, al contrario de lo que se había asumido hasta el momento, a saber, el protagonismo de Rabindranath Tagore, Abanindranath Tagore o incluso de Ordhendra Coomar Gangoly.

11 Véase también la fuente "Die Schöpfung, Rigveda X" (Adler, 1921) y la portada del libro: Margit Téry-Adler, Utopia. Dokumente der Wirklichkeit, litografía en color, 33 × 24,5 cm, Bauhaus-Archiv Berlin (Bittner/Rhomberg, 2013: 39).

12 Abanindranath Tagore y Gaganendranath Tagore eran sobrinos segundos del poeta Rabindranath Tagore aunque prácticamente de la misma generación. Sobre la obra plástica de los tres véase la monografía de Parimoo (2011).

13 K. Manjapra sugiere esta idea: «The works of the German Bauhaus were not brought to Calcutta in order to be fetishized as exceptional, but to be compared with contemporary Indian artworks» (Manjapra, 2011: 36).

14 Traducción del original (todas las traducciones del inglés y del alemán han sido realizadas por la autora): «The Indian public should study this exhibition for then, they may learn that European Art does not mean "naturalism" and that the transformation of the forms of nature in the work of an artist is common to ancient and modern India and Europe as an unconscious and therefore inevitable expression of the life of soul and artistic genius".

15 P. Mitter declara: «We may take December 1922 as a convenient entry point for modernism in India. An exhibition of works of the Bauhaus artists in Calcutta in that year symbolized the graduation of Indian taste from Victorian naturalism to non-representational art» (Mitter, 2007: 15).

16 Benoy Kumar Sarkar, fue un sociólogo y economista indio que residió largo tiempo en Europa. Tras la Primera Guerra Mundial se dedicó a coordinar las actividades de la diáspora india en Berlín (Manjapra, 2013: 137-147).

17 Del original en alemán: «nationalem Selbstbewusstsein».

18 Del original en alemán: «eine gewisse geistige Einigkeit die gesamte moderne Kunstwelt organisch vebindet».

19 Benoy Kumar Sarkar visitó en torno a 1920 el Institut für Seeverkehr und Weltwirtschaft de Kiel que, fundado por el economista alemán Bernhard Harms, se constituyó como centro de investigación para el internacionalismo económico alemán durante las décadas de 1910 y 1920. Sarkar y Harms compartían el afán por impulsar los intercambios comerciales de sus países de origen con otras potencias extranjeras y ambos estaban interesados las teorías comerciales e interpretaciones del nuevo orden capitalista que debía instituirse más allá de la hegemonía del Imperio británico (Manjapra 2014: 149-155).

20 Se reproduce la cita completa de B. Kumar Sarkar: «lt is now time to cast aside the negatives and enunciate our position in as positive a manner as possible. What are we to understand by the emancipation, that is being advocated here, of art from the despotism of literary criticism, historical or philosophical analysis, ethical or religious studies, and democratic, bolshevistic or nationalistic propaganda? What is the meaning of the thesis that we should have to conceive shilpa as a svaraja in itself, i.e., to treat art or creation of "beauties" as a self-determined entity in human experience? In what sense it is possible to concede to painting and sculpture an absolute autonomy, whether as modes of objective description or subjective expression?» (Kumar Sarkar, 1922: 16). Ya P. Mitter hace referencia 
a la comparación realizada por Benoy Kumar Sarkar entre la demanda nacionalista de autogobierno en India y la noción vanguardista de autonomía estética (Mitter, 2007: 16).

El término shilpa, literalmente adorno o trabajo manual, denomina en principio el trabajo artístico que implica habilidad técnica pero no actividad intelectual, y cuyo fin no apela a los sentidos sino a su utilidad. Mientras el término kalā hace referencia al arte en general, ya sea pintura, escultura o artes escénicas. Ananda K. Coomaraswamy identificó en sus escritos kalā con las artes vocacionales y shilpa con la artesanía, tal vez tratando de equipararlas a la distinción occidental entre Bellas Artes y Artes Decorativas. Sin embargo, en sus últimos ensayos destacó la importancia del arte para la vida cotidiana y afirmó que en India es imposible la distinción entre artes populares o decorativas y Bellas Artes, idea que fue asimismo fundamental en el desarrollo de la modernidad india, especialmente en la experimentación realizada en la escuela de arte Kala-Bhavana en Sāntiniketan (Fernández del Campo, 2013: 18-28). Por lo tanto, en el texto de Sarkar, el concepto shilpa asociado al swarāj se refiere a la creación artística según la concepción tradicional india, donde no existe distinción entre artes mayores y menores.

El término svaraja o swarāj traducido al inglés como self-rule o self-governance [autogobierno], se refiere al concepto promulgado por Mahatma Gandhi para apelar a la independencia india del yugo colonial británico. La principal estrategia de este movimiento fue el denominado Swadeshi, la autosuficiencia económica a través del boicot de los productos ingleses y la recuperación de las técnicas y productos tradicionales indios. Sobre la relación entre los movimientos artísticos modernos, la recuperación de las técnicas tradicionales y la estrategia Swadeshi véase los escritos de Coomaraswamy sobre el tema (Coomaraswamy 1994: 1-29).

21 Del original en inglés: "Try to believe in the regeneration of India through art, and not by politics and economics alone».

22 Véase la cita de Kramrisch: «Ultimately all art results from the union of intuition and personality. The first universal, unlimited and unchangeable consists in intensity and tends towards expression, the latter confined in temporal, national and individual limits enables the variety of visualisations, the various designs or compositions. Every living, i. e., creative art has in itself a necessity of composition endowed with a melody and logic of its own". (Kramrisch, 1922a: 66-67).

23 Rabindranath Tagore fue crítico con el arte de la Escuela de Bengala por su aislamiento elitista y tradicional, lo que iba contra su intención de crear lazos con las corrientes internacionales y su interés por las artes populares. (Bittner/Rhomberg, 2013: 100).

24 Acerca del desarrollo de la escuela de arte Kala-Bhavana en Śāntiniketan y concretamente sobre los intercambios tanto con Oriente como con Occidente, véase los artículos y tesis doctoral de S. Román Aliste (2013a: 187-796; 2013b; 2015).

25 Si se toma, por ejemplo, el periódico Die Voss/Vossische Zeitung (véase nota siguiente sobre este periódico) entre los años 1921 y 1923 se encuentran numerosas referencias a Rabindranath Tagore (véase los artículos de 1921; Kämpchen, 2011).

26 El Vossische Zeitung fue un periódico liberal burgués publicado en Berlín, desde 1617 bajo el nombre Voss / Vossische y desde 1910 Vossiche Zeitung, su última tirada fue en 1934, por motivo de la censura del régimen nacionalsocialista. Durante la República de Weimar tuvo gran repercusión, no sólo en Berlín sino también en otros territorios alemanes.

27 Cita completa en alemán: «Was sich vor uns ausbreitet, sind Dokumente eines grossartigen kulturellen Befreiungskampfes, der sich gegen einen übermächtigen Druck auflehnt».

28 Del original en alemán: «Gogonendra Nath Tagore, scheint gar kubistische Anregungen in sich aufgenommen zu haben. Das Schwarzweiß-Blatt eines „Tempels“ lässt Asiatisches mit den kristallinischen Formen zusammenklingen, wie sie etwa Feininger gern verwendet. Die reizende, buntschillernde Farbenphantasie „Im Vogellande“ will zu Franz Marc hinübergerufen».

29 Traducido de la publicación en inglés: «The world-wide yearning for a grand expression is evident here - the yearning which to-day has taken hold of all artists of all countries and races".

30 Véase la cita completa: «The relation of Indian and European art was, until the present time, either based on an inner affinity or it resulted from a connection of trade and from similar conditions of culture. The transplantation however of European tradition into Indian art schools is artificial and useless. But art despises all purposes; it is as closely connected with life as a child is connected with its mother. Foreign children can never become her own. Still one feature of the present age must not be overlooked. Not only Indian art, but first of all the Indian outlook, has deep effect on modern Western spiritually, while at the same time the East accepts European civilization. Whatever the result may be this exchange means movement. Movement is a sign of life, and life is productive, whether it is in the purposed sense or by contradiction and reaction, makes no difference» (Kramrisch, 1922b). Respecto a las relaciones entre la pintura india contemporánea y la vanguardia occidental, véase también el artículo de Kramrisch (1924: 234-242).

31 Del original en inglés: "Cubism was a "discovery" of the West. The roots of it however lie in the East. "Backgrounds" in Ajanta, many objects represented in Bharut and Sanchi, the architecture in Rajput paintings are visualised [sic.] in cubist way. Modern European art, tired of reproduction, set forth to rediscover the expressive suggestiveness of the primary elements of art, of line and cube and their relations, of the colours and their life, and of light and shade".

32 Del original en inglés: "The crux of the whole question lies in an intellectual and spiritual synthesis of Eastern and Western culture in which the question of "influence" is an irrelevant and misleading topic. The word "influence" inevitably suggest a domination or the supremacy of one over the other".

\section{Bibliografía}

(1921) «Rabindranath Tagore - Fremde Gäste an deutschen Hochschulen», Die Voss, n. ${ }^{\circ}$ 19, Samstag, 11, junio.

(1921) «Rabindranath Tagore über indische Kultur», Vossische Zeitung, n. 236 (Sonntags-Ausgabe), Sonntag, 22, mayo.

(1922) «European Influence on Modern Indian Art», Rupam, n. ${ }^{\circ} 11$, julio, p. 109.

(1923) «The Fourteenth Annual Exhibition of the Indian Society of Oriental Art», Rupam, n. ${ }^{\circ} 13-14$, enero-junio, pp. 14-18.

(1923) «The Indian Art Exhibition: Cuttings from German Press», Rupam, n. 15-16, julio-diciembre, pp. 78-83.

ADLER, Bruno (ed.) (1921), Utopia. Dokumente der Wirklichkeit, Weimar. 
BHABHA, Homi K. (1994), The Location of Culture, Routledge, Londres, Nueva York.

BITTNER, Regina y RHOMBERG, Kathrin (2013), "Das Bauhaus in Kalkutta. Weltkunst seit 1922. Zur Aktualität einer Ausstellung», en Das

Bauhaus in Kalkutta. Eine Begegnung kosmopolitischer Avantgarden, Ostfildern, pp. 65-84.

- (eds.) (2013), Das Bauhaus in Kalkutta. Eine Begegnung kosmopolitischer Avantgarden, Hatje Cantz Verlag, Ostfildern.

COOMARASWAMY, Ananda Kentish (1994), Art and Swadeshi, Nueva Delhi.

FERNÁNDEZ DEL CAMPO, Eva (2013), Cánones del arte indio, Abada, Madrid.

FOSTER, Hall (ed.) (2004), Art since 1900. Modernism, Antimodernism, Postmodernism, Thames \& Hudson, Londres.

FRIEDEWALD, Boris (2013), «Das Bauhaus und Indien - Blick zurück in die Zukunft», en BITTNER, Regina y RHOMBERG, Kathrin (eds.), Das

Bauhaus in Kalkutta. Eine Begegnung kosmopolitischer Avantgarden, Hatje Cantz Verlag, Ostfildern, pp. 119-133.

GUHA-THAKURTA, Tapati (1992), The Making of a New «Indian» Art: Artists, Aesthetics and Nationalism in Bengal 1850-1920, Cambridge

University Press, Cambridge.

JANDA, Annegret y GRABOWSKI, Jörn (eds.) (1992), Kunst in Deutschland 1905-1937. Die verlorene Sammlung der Nationalgalerie im ehemaligen Kronprinzen-Palais: Dokumentation, Staatliche Museen zu Berlin - Preussischer Kulturbesitz, Berlín.

KÄMPCHEN, Martin (2011) Rabindranāth Tagore und Deutschland, Deutsche Schillergesellschaft, Marbach am Neckar.

KRAMRISCH, Stella (1922a), "The Aesthetics of a Young India. A Rejoinder», Rupam, n. 10, abril, pp. 66-67.

- (1922b), «Indian Art and Europe», Rupam, n. ${ }^{\circ} 11$, julio, pp. 85-86.

- (1922c), «An Indian Cubist», Rupam, n. ${ }^{\circ} 11$, julio, p. 108.

- (1922d), Catalogue of The Fourteenth Annual Exhibition of the Indian Society of Oriental Art, December 1922, en BITTNER, Regina y

RHOMBERG, Kathrin (eds.) (2013), Das Bauhaus in Kalkutta. Eine Begegnung kosmopolitischer Avantgarden, Hatje Cantz Verlag,

Ostfildern, pp. 11-14.

- (1924), «Indische Malerei der Gegewart», Jahrbuch der jungen Kunst, vol. 4, pp. 234-242.

KUMAR SARKAR, Benoy (1922), «The Aesthethics of a Young India», Rupam, n. 9, enero, pp. 8-24.

- (1923), Ausstellung moderner indischer Aquarelle in der Nationalgalerie, Indo-Europäische Handelsgesellschaft, Berlín.

MANJAPRA, Kris (2011), "Stella Kramrisch and the Bauhaus in Calcutta», en SIVA KUMAR, Raman (ed.), The Last Harvest. Paintings of Rabindranath Tagore, Mapin Publishing, Ahmedabad, pp. 34-40.

- (2013), «Das antikoloniale Labor. Die indisch-nationale Diaspora im deutschsprachigen Europa», en BITTNER, Regina y RHOMBERG,

Kathrin (eds.), Das Bauhaus in Kalkutta. Eine Begegnung kosmopolitischer Avantgarden, Hatje Cantz Verlag, Ostfildern, pp. $137-147$.

- (2014), Age of Entanglement. German and Indian Intellectuals Across Empire, Harvard University Press, Cambridge, Massachusetts, Londres.

MITTER, Partha (1994), Art and Nationalism in Colonial India 1850-1922, Cambridge University Press, Cambridge.

- (2007), The Triumph of Modernism. India's artists and the avant-garde 1922-1947, Reaktion Books, Londres.

- (2008), «Decentering Modernism. Art History and Avant-Garde Art from the Periphery», The Art Bulletin, vol. 90, n. ${ }^{\circ}$ 4, pp. $531-548$.

- (2013), «Moderne, Kunst und nationale Identität in Indien. Hintergrund zur Bauhausausstellung in Kalkutta 1922», en BITTNER, Regina y

RHOMBERG, Kathrin (eds.), Das Bauhaus in Kalkutta. Eine Begegnung kosmopolitischer Avantgarden, Hatje Cantz Verlag, Ostfildern, pp. 85-93.

OESTERHELD, Joachim (2014), «Germans in India Between Kaiserreich and the end of World War Il», en MIYANG CHO, Joanne; KURLANDER, Eric y MCGETCHIN, Douglas (eds.), Transcultural Encounters Between Germany and India. Kindred Spirits in the Nineteenth and

Twentieth Centuries, Routledge, Londres, pp. 101-114.

OSBORN, Max (1923a), «Indische Kunst in Berlin», Die Voss, Wochen-Auslands-Ausgabe, n. ${ }^{\circ}$ 10, Sonnabend, 10 , marzo.

- (1923b) «Moderne indische Kunst. Ausstellung im Kronprinzenpalais», Vossische Zeitung, n. 63 (Morgen-Ausgabe), Erste Beilage zur

Vossischen Zeitung, Mittwoch, 7, febrero, p. 1.

- (1923c), «The Indian Art Exhibition in Berlin», Rupam, n. ${ }^{\circ}$ 15-16, julio-diciembre, p. 77.

PARIMOO, Ratan (2011), Art of the Three Tagores. From Revival to Modernity, Kumar Gallery, Nueva Delhi. 
ROMÁN ALISTE, Sergio (2013), «El proyecto de pedagogía artística de Śāntiniketan como encrucijada de la integración de arte y vida en la India Contemporánea (1920-1950)", comunicación en el V Encuentro de Jóvenes Investigadores Complutense, Historia del Arte, Facultad de Geografía e Historia de la Universidad Complutense de Madrid, 6-7 mayo 2013 (próxima publicación en la revista Anales de Historia del Arte, cedido por el autor).

- (2013), «La enseñanza del arte en Śāntiniketan como punto de encuentro de Japón y Europa bajo el amparo ideológico de Rabindranath Tagore», en P. GARCÉS GARCÍA y L. TERRÓN BARBOSA (eds.), Itinerarios, viajes y contactos Japón-Europa, Peter Lang, Berna, pp. 787-796.

- (2015), Dimensiones artísticas de la pedagogía en Śāntiniketan. Un proyecto internacional en el medio rural bengalí (1901-1951), Tesis Doctoral, Universidad Complutense de Madrid.

STOLER MILLER, Barbara (1983), Exploring India's sacred art: selected writings of Stella Kramrisch, Motilal Banarsidass Publishe, Filadelfia.

STRUVE, Karen (2013), «Phänomene - Hybridität und Dritter Raum», en Zur Aktualität von Homi K. Bhabha. Einleitung in sein Werk, Springer, Wiesbaden, pp. 97-117.

WAGNER, Christoph (2005), «Zwischen Lebensreform und Esoterik: Johannes Ittens Weg ans Bauhaus Weimar», en Johannes Itten - Wassily Kandinsky - Paul Klee. Das Bauhaus un die Esoterik, Bielefeld, pp. 65-75.

WINKLER, Kurt (2002), Museum und Avantgarde. Ludwig Justis Zeitschrift «Museum der Gegenwart» und die Musealisierung des Expressionismus, Berliner Schriften zur Museumskunde, Band 17, Institut für Museumskunde, Staatliche Museen zu Berlin - Preussischer Kulturbesitz, Oplanden. 
